\title{
A FUNDAMENTAL THEOREM FOR SUBMANIFOLDS OF MULTIPRODUCTS OF REAL SPACE FORMS
}

\author{
MARIE-AMÉLIE LAWN AND JULIEN ROTH
}

\begin{abstract}
We prove a Bonnet theorem for isometric immersions of submanifolds into the products of an arbitrary number of simply connected real space forms. Then, we prove the existence of associated families of minimal surfaces in such products. Finally, in the case of $\mathbb{S}^{2} \times \mathbb{S}^{2}$, we give a complex version of the main theorem in terms of the two canonical complex structures of $\mathbb{S}^{2} \times \mathbb{S}^{2}$.
\end{abstract}

\section{INTRODUCTION}

It is a classical problem of submanifold theory to determine when a Riemannian manifold $\left(M^{n}, g\right)$ can be immersed into a fixed Riemannian manifold $\left(\bar{M}^{n+p}, \bar{g}\right)$. The well-known Gauss, Ricci and Codazzi equations relate the intrinsic and extrinsic curvatures, and any submanifold of any Riemannian manifold must satisfy them. Conversely, the classical Bonnet theorem [1] states that on a surface, given first and second fundamental forms satisfying the Gauss and Codazzi equations, this surface is locally embeddable into the Euclidean 3 -space $\mathbb{R}^{3}$. This result can be generalized to higher codimension [10, and the classical Fundamental Theorem of Submanifolds states that, in fact, the Gauss, Codazzi and Ricci equations are necessary and sufficient conditions for a Riemannian $n$-dimensional manifold to admit a (local) immersion into a space of constant sectional curvature of dimension $n+d$.

If the ambient space is not of constant sectional curvature, proving fundamental theorems is technically difficult and there are few results known. Moreover, the Gauss, Codazzi and Ricci equations are in general not sufficient anymore and other conditions are required in order to produce the immersion. In [3, Daniel gave such a characterization for surfaces in the three-dimensional Thurston geometries with four-dimensional isometry groups, by computing the Christoffel symbols explicitly and using the technique of Cartan moving frames. In higher dimensions, he also stated in 2 necessary and sufficient conditions for an $n$-dimensional Riemannian manifold to be isometrically immersible into the products $\mathbb{S}^{n} \times \mathbb{R}$ and $\mathbb{H}^{n} \times \mathbb{R}$, also using the moving frame technique. This allowed him to study the existence of associated families in the case of minimal surfaces. This result was later generalized by the second author $[9$ in the case where the ambient space is a Lorentzian product. Very recently, Ortega and the first author [7] proved fundamental theorems characterizing immersions of hypersurfaces into (quasi-)Einstein manifolds, specifically Robertson-Walker warped products. These spaces play an important role in standard models of cosmology, arising as solutions of the non-vacuum Einstein equations, and have therefore a great importance in Lorentzian geometry. As an application, conditions were obtained for 3-dimensional hypersurfaces in Robertson-Walker spacetimes to be foliated by surfaces whose mean curvature vector is either lightlike or zero (including maximal surfaces, marginally outer

2010 Mathematics Subject Classification. 53A42, 53C20, 53C21.

Key words and phrases. Almost-umbilical hypersurfaces, space forms, constant scalar curvature. 
trapped surfaces (MOTS), and mixed cases), hence providing an helpful tool for the study of horizons on Robertson-Walker spacetimes with spacelike or timelike causal character, including marginally outer trapped tubes.

Extending the result of [2], Kowalczyk and Lira-Tojeiro-Vitório proved independently in [6] and 8] the existence and uniqueness of isometric immersions in a product of two spaces forms of constant sectional curvature. In this paper we generalize their result to immersions into multiproducts $\tilde{P}=M_{1} \times \cdots \times M_{m}$ of real space form of arbitrary dimension and arbitrary sectional curvature. The key idea is to use the projections $\pi_{i}, i \in 1, \ldots, m$ into each of the factors of the product. Each projection induces then two operators on the tangent bundle and two operators on the normal bundle of the submanifold satisfying some properties and some compatibility equations which can be deduced from the Gauss and Weingarten formulas of the immersion. We prove that, conversely, these conditions together with the Gauss, Codazzi and Ricci equations are necessary and sufficient conditions to immerse a Riemannian manifold isometrically into such an ambient space.

As an application we then prove the existence of a one-parameter associated family of isometric immersions for minimal surfaces in multiproducts. Finally we consider the special case where the ambient space is $\mathbb{S}^{2} \times \mathbb{S}^{2}$ and give a complex version of our fundamental theorem in terms of the induced complex structures.

The authors want to thank F. Torralbo for helpful discussions.

\section{Multiproducts of SPACE FORMS AND THEIR SUBMANIFOLDS}

We consider the product space $\left(\widetilde{P}=M_{1} \times \cdots \times M_{m}, \widetilde{g}=g_{1} \oplus \cdots \oplus g_{m}\right)$ where $\left(M_{i}, g_{i}\right)$ is the simply connected real space form of dimension $n_{i}$ and constant sectional curvature $c_{i}$. Moreover, without loss of generality, we assume that $c_{i} \neq 0$ for $i \in\{1, \cdots, m-1\}$ and that $c_{m}$ may possibly be zero. We denote by $\pi_{i}$ the projection of any tangent vector $X$ on $T M_{k}$. These projections satisfy the following relations

$$
\left\{\begin{array}{l}
\widetilde{g}\left(\pi_{i} X, Y\right)=\widetilde{g}\left(X, \pi_{i} Y\right) \text { for any } X, Y \in \Gamma(T P) \\
\pi_{i} \circ \pi_{i}=\pi_{i} \\
\pi_{i} \circ \pi_{j}=0 \text { if } i \neq j \\
\widetilde{\nabla} \pi_{i}=0 \\
\operatorname{rank}\left(\pi_{i}\right)=n_{i} \\
\sum_{i=1}^{m} \pi_{i}=\operatorname{Id}_{T \widetilde{P}}
\end{array}\right.
$$

Moreover, the curvature tensor of $\widetilde{P}$ is given by

$$
\widetilde{R}(X, Y) Z=\sum_{i=1}^{m} c_{i}\left[\left\langle\pi_{i} Y, \pi_{i} Z\right\rangle \pi_{i} X-\left\langle\pi_{i} X, \pi_{i} Z\right\rangle \pi_{i} Y\right] .
$$

Now, we consider a Riemannian manifold $\left(M^{n}, g\right)$ isometrically immersed into $\widetilde{P}$. We denote by $N M$ the normal bundle and by $\nabla^{\perp}$ the normal connection and by $B: T M \times T M \longrightarrow N M$ the second fundamental form. For any $\xi \in N M, A_{\xi}$ is the Weingarten operator associated to $\xi$ and defined by $\widetilde{g}\left(A_{\xi} X, Y\right)=\widetilde{g}(B(X, Y), \xi)$, with $X, Y$ vectors tangent to $M$.

For any $i \in\{1, \cdots, m\}$, the projection $\pi_{i}$ induces the existence of the following four operators $f_{i}: T M \rightarrow T M, h_{i}: T M \rightarrow N M, s_{i}: N M \rightarrow T M$ and $t_{i}: N M \rightarrow N M$, such that

$$
\pi_{i} X=f_{i} X+h_{i} X \quad \text { and } \quad \pi_{i} \xi=s_{i} \xi+t_{i} \xi .
$$


From the symmetry of the $\pi_{i}$, we obtain that, for any $i \in\{1, \cdots, m\}, f_{i}$ and $t_{i}$ are symmetric and for any $X \in \Gamma(T M)$ and $\xi \in \Gamma(N M)$

$$
\widetilde{g}\left(h_{i} X, \xi\right)=\widetilde{g}\left(X, s_{i} \xi\right)
$$

In addition, from the fact that $\sum_{i=1}^{m} \pi_{i}=I d_{T \widetilde{P}}$, we get the following identities

$$
\sum_{i=1}^{m} f_{i}=I d_{T M}, \quad \sum_{i=1}^{m} t_{i}=I d_{E}, \quad \sum_{i=1}^{m} s_{i}=0 \quad \text { and } \quad \sum_{i=1}^{m} h_{i}=0 .
$$

Moreover, we have the following relations between these operators coming from the fact that $\pi_{i} \circ \pi_{j}=\delta_{i}^{j} \pi_{i}$

$$
\begin{aligned}
& f_{i} \circ f_{j}+s_{i} \circ h_{j}=\delta_{i}^{j} f_{i}, \\
& t_{i} \circ t_{j}+h_{i} \circ s_{j}=\delta_{i}^{j} t_{i}, \\
& f_{i} \circ s_{j}+s_{i} \circ t_{j}=\delta_{i}^{j} s_{i}, \\
& h_{i} \circ f_{j}+t_{i} \circ h_{j}=\delta_{i}^{j} h_{i},
\end{aligned}
$$

where $\delta_{i}^{j}$ is the classical Kronecker symbol, that is, 1 if $i=j$ and 0 if $i \neq j$. Moreover, from the fact that $\pi_{i}$ is parallel, we deduce easily that for any $X, Y \in T M$ and $\xi \in N M$, we have

$$
\begin{aligned}
& \nabla_{X}\left(f_{i} Y\right)-f_{i}\left(\nabla_{X} Y\right)=A_{h_{i} Y} X+s_{i}(B(X, Y)), \\
& \nabla_{X}^{\perp}\left(h_{i} Y\right)-h_{i}\left(\nabla_{X} Y\right)=t_{i}(B(X, Y))-B\left(X, f_{i} Y\right), \\
& \nabla_{X}^{\perp}\left(t_{i} \xi\right)-t_{i}\left(\nabla_{X}^{\perp} \xi\right)=-B\left(s_{i} \xi, X\right)-h_{i}\left(A_{\xi} X\right), \\
& \nabla_{X}\left(s_{i} \xi\right)-s_{i}\left(\nabla_{X}^{\perp} \xi\right)=-f_{i}\left(A_{\xi} X\right)+A_{t_{i} \xi} X .
\end{aligned}
$$

Finally, from the expression for the curvature tensor $\widetilde{R}$, we get the following Gauss, Codazzi and Ricci equations

$$
\begin{gathered}
R(X, Y) Z=\sum_{i=1}^{m} c_{i}\left[\left\langle f_{i} Y, Z\right\rangle f_{i} X-\left\langle f_{i} X, Z\right\rangle f_{i} Y\right]+A_{B(Y, Z)} X-A_{B(X, Z)} Y \\
\left(\nabla_{X} B\right)(Y, Z)-\left(\nabla_{Y} B\right)(X, Z)=\sum_{i=1}^{m} c_{i}\left[\left\langle f_{i} Y, Z\right\rangle h_{i} X-\left\langle f_{i} X, Z\right\rangle h_{i} Y\right], \\
R^{\perp}(X, Y) \xi=\sum_{i=1}^{m} c_{i}\left[\left\langle h_{i} Y, \xi\right\rangle h_{i} X-\left\langle h_{i} X, \xi\right\rangle h_{i} Y\right]+B\left(A_{\xi} Y, X\right)-B\left(A_{\xi} X, Y\right) .
\end{gathered}
$$

\section{MAin RESUlt}

Now, conversely, consider $\left(M^{n}, g\right)$ a Riemannian manifold and $E$ a $d$-dimensional vector bundle over $M$ endowed with a metric $\bar{g}$ and a compatible connection $\bar{\nabla}$. Moreover, let $B: T M \times$ $T M \longrightarrow E$ be a symmetric (2,1)-tensor and $f_{i}: T M \longrightarrow T M, h_{i}: T M \longrightarrow E$ and $t_{i}: E \longrightarrow E$ be some (1,1)-tensors for $i \in\{1, \cdots, m\}$. We define $s_{i}$ as the dual of $h_{i}$ with respect to the metrics $\widetilde{g}:=g \oplus \bar{g}$ on $T M \oplus E$, that is, for any $X \in T_{x} M$ and $\xi \in E_{x}$,

$$
\bar{g}_{x}\left(h_{i} X, \xi\right)=g_{x}\left(X, s_{i} \xi\right)
$$


Finally, for any $\xi \in \Gamma(E)$, we define $A_{\xi}$ by

$$
\left\langle A_{\xi} X, Y\right\rangle=\langle B(X, Y), \xi\rangle,
$$

for any $X, Y \in \Gamma(T M)$. Following the discussions of Section 2, we introduce now the following natural definition.

Definition 3.1. We say that $\left(M, g, E, \bar{g}, \bar{\nabla}, B, f_{i}, h_{i}, t_{i}\right)$ satisfies the compatibility equations for the multiproduct $\widetilde{P}=M_{1} \times \cdots \times M_{m}$ if

i) $f_{i}$ and $t_{i}$ are symmetric for any $i \in\{1, \cdots, m\}$,

ii) for any $i \in\{1, \cdots, m\}$, Equations (44)-(11) are satisfied, that is,

$$
\begin{aligned}
& \sum_{i=1}^{m} f_{i}=I d_{T M}, \quad \sum_{i=1}^{m} t_{i}=I d_{E}, \quad \sum_{i=1}^{m} s_{i}=0 \quad \text { and } \quad \sum_{i=1}^{m} h_{i}=0 \\
& f_{i} \circ f_{j}+s_{i} \circ h_{j}=\delta_{i}^{j} f_{i}, \\
& t_{i} \circ t_{j}+h_{i} \circ s_{j}=\delta_{i}^{j} t_{i}, \\
& f_{i} \circ s_{j}+s_{i} \circ t_{j}=\delta_{i}^{j} s_{i}, \\
& h_{i} \circ f_{j}+t_{i} \circ h_{j}=\delta_{i}^{j} h_{i}, \\
& \nabla_{X}\left(f_{i} Y\right)-f_{i}\left(\nabla_{X} Y\right)=A_{h_{i} Y} X+s_{i}(B(X, Y)), \\
& \bar{\nabla}_{X}\left(h_{i} Y\right)-h_{i}\left(\nabla_{X} Y\right)=t_{i}(B(X, Y))-B\left(X, f_{i} Y\right), \\
& \bar{\nabla}_{X}\left(t_{i} \xi\right)-t_{i}\left(\bar{\nabla}_{X} \xi\right)=-B\left(s_{i} \xi, X\right)-h_{i}\left(A_{\xi} X\right),
\end{aligned}
$$

iii) the rank of $\pi_{i}$ is $n_{i}$ for any $i \in\{1, \cdots, m\}$ and $\sum_{i=1}^{m} n_{i}=n+p$, and

iv) the Gauss, Ricci and Codazzi equations (G), (C) and $(\underline{\mathrm{R}})$ are satisfied. Namely for any $X, Y, Z \in \Gamma(T M)$ and any $\xi \in \Gamma(E)$,

$$
\begin{aligned}
& R(X, Y) Z=\sum_{i=1}^{m} c_{i}\left[\left\langle f_{i} Y, Z\right\rangle f_{i} X-\left\langle f_{i} X, Z\right\rangle f_{i} Y\right]+A_{B(Y, Z)} X-A_{B(X, Z)} Y, \\
& \left(\nabla_{X} B\right)(Y, Z)-\left(\nabla_{Y} B\right)(X, Z)=\sum_{i=1}^{m} c_{i}\left[\left\langle f_{i} Y, Z\right\rangle h_{i} X-\left\langle f_{i} X, Z\right\rangle h_{i} Y\right], \\
& \bar{R}(X, Y) \xi=\sum_{i=1}^{m} c_{i}\left[\left\langle h_{i} Y, \xi\right\rangle h_{i} X-\left\langle h_{i} X, \xi\right\rangle h_{i} Y\right]+B\left(A_{\xi} Y, X\right)-B\left(A_{\xi} X, Y\right),
\end{aligned}
$$

where $\bar{R}$ is the curvature associated with the connection $\bar{\nabla}$.

We can now state the main result of the paper.

Theorem 3.2. Let $\left(M^{n}, g\right)$ be a simply connected Riemannian manifold and $E$ a d-dimensional vector bundle over $M$ endowed with a metric $\bar{g}$ and a compatible connection $\nabla^{\perp}$. Moreover, let $B: T M \times T M \longrightarrow E$ be a symmetric $(2,1)$-tensor and $f_{i}: T M \longrightarrow T M, h_{i}: T M \longrightarrow E$ and $t_{i}: E \longrightarrow E$ be some $(1,1)$-tensors for $i \in\{1, \cdots, m\}$. If $\left(M, g, E, \bar{g}, \bar{\nabla}, B, f_{i}, h_{i}, t_{i}\right)$ satisfies the compatibility equations for the multiproduct $\widetilde{P}=M_{1} \times \cdots \times M_{m}$ then, there exists an isometric immersion $\varphi: M \longrightarrow \widetilde{P}$ such that the normal bundle of $M$ for this immersion is isomorphic to $E$ and such that the second fundamental form, the normal connection and the projections on each factor of $T \widetilde{P}_{\mid M}$ are given by $B, \bar{\nabla}$ and $\left(f_{i}, h_{i}, t_{i}\right)$ respectively. Precisely, there exists a vector bundle isometry $\widetilde{\varphi}: E \longrightarrow(\varphi(M))^{\perp}$ so that

$$
\pi_{i}\left(\varphi_{*} X\right)=\varphi_{*}\left(f_{i} X\right)+\Phi\left(h_{i} X\right)
$$




$$
\begin{gathered}
\pi_{i}(\widetilde{\varphi} \xi)=\varphi_{*}\left(s_{i} X\right)+\Phi\left(t_{i} \xi\right), \\
I I_{f}=\widetilde{\varphi} \circ B, \\
\nabla^{\perp} \widetilde{\varphi}=\widetilde{\varphi} \bar{\nabla} .
\end{gathered}
$$

Moreover, this isometric immersion is unique up to an isometry of $\widetilde{P}$.

Our approach to prove this theorem is not based on the moving frame technique, but is in the spirit of [6] and uses techniques introduced in [4] and [5].

Proof: We give the proof for the case $c_{m} \neq 0$, the case $c_{m}=0$ can be proved analogously with minor changes. First, for any $i \in\{1, \ldots, m\}$, let us denote by $E_{i}$ a trivial line bundle over $M$ equipped with the Euclidean metric, if $c_{i}>0$, and minus the Euclidean metric, if $c_{i}<0$. We consider the vector bundle $F$ over M,

$$
F=T M \oplus E \underset{i=1}{\oplus} E_{i}
$$

defined by the orthogonal Withney sum of Riemannian vector bundles. We denote by $\widetilde{g}$ the metric over $F$ obtained from $g, \bar{g}$ and the metrics on each $E_{i}$. For any $i \in\{1, \ldots, m\}$, we consider a section $\xi_{i}$ of $E_{i}$ such that $\widetilde{g}\left(\xi_{i}, \xi_{i}\right)=\frac{1}{c_{i}}$. We introduce now the following connection on $F$, denoted by $D$

$$
\begin{aligned}
& D_{X} Y=\nabla_{X} Y+B(X, Y)-\sum_{i=1}^{m} c_{i} g\left(f_{i} X, Y\right) \xi_{i}, \\
& D_{X} \nu=\nabla_{X}^{\perp} \nu-A_{\nu} X-\sum_{i=1}^{m} c_{i} \bar{g}\left(h_{i} X, \nu\right) \xi_{i}, \\
& D_{X} \xi_{i}=f_{i} X+h_{i} X,
\end{aligned}
$$

for any vector fields $X, Y$ tangent to $M$ and any section $\nu$ of $E$.

Lemma 3.3. The connection $D$ is compatible with the metric $\widetilde{g}$.

Proof: This comes easily from the definition. Let $X, Y, Z \in \Gamma(T M), \nu, \eta \in \Gamma(E)$. We have

$$
\begin{aligned}
X \widetilde{g}(Y, Z) & =X g(Y, Z) \\
& =g\left(\nabla_{X} Y, Z\right)+g\left(Y, \nabla_{X} Z\right) \\
& =\widetilde{g}\left(D_{X} Y, Z\right)+\widetilde{g}\left(Y, D_{X} Z\right),
\end{aligned}
$$

since the tangential parts of $D_{X} Y$ and $D_{X} Z$ are $\nabla_{X} Y$ and $\nabla_{X} Z$ respectively. Similarly, we have

$$
\begin{aligned}
X \widetilde{g}(\nu, \eta) & =X \bar{g}(\nu, \eta) \\
& =\bar{g}\left(\nabla_{X}^{\perp} \nu, \eta\right)+\bar{g}\left(\nu, \nabla_{X}^{\perp} \eta\right) \\
& =\widetilde{g}\left(D_{X} \nu, \eta\right)+\widetilde{g}\left(\nu, D_{X} \eta\right),
\end{aligned}
$$

since the normal parts of $D_{X} \nu$ and $D_{X} \eta$ are $\nabla_{X}^{\perp} \nu$ and $\nabla_{X}^{\frac{1}{X}} \eta$ respectively. Moreover, we have

$$
\begin{aligned}
X \widetilde{g}\left(\xi_{i}, \xi_{j}\right) & =0 \\
& =\widetilde{g}\left(f_{i} X+h_{i} X, \xi_{j}\right)+\widetilde{g}\left(\xi_{i}, f_{j} X+h_{j} X\right) \\
& =\widetilde{g}\left(D_{X} \xi_{i}, \xi_{j}\right)+\widetilde{g}\left(\xi_{i}, D_{X} \xi_{j}\right) .
\end{aligned}
$$


Finally for mixed terms, we have

$$
\begin{aligned}
X \widetilde{g}\left(\xi_{i}, Y\right) & =0 \\
& =g\left(f_{i} X, Y\right)-c_{i} \widetilde{g}\left(\xi_{i}, \xi_{i}\right) g\left(f_{i} X, Y\right) \\
& =\widetilde{g}\left(f_{i} X+h_{i} X, Y\right)+\widetilde{g}\left(\xi_{i}, \nabla_{X} Y+B(X, Y)-\sum_{i=1}^{m} c_{i} g\left(f_{i} X, Y\right) \xi_{i}\right) \\
& =\widetilde{g}\left(D_{X} \xi_{i}, \xi_{j}\right)+\widetilde{g}\left(\xi_{i}, D_{X} \xi_{j}\right),
\end{aligned}
$$

and

$$
\begin{aligned}
X \widetilde{g}\left(\xi_{i}, \nu\right) & =0 \\
& =g\left(h_{i} X, \nu\right)-c_{i} \widetilde{g}\left(\xi_{i}, \xi_{i}\right) g\left(h_{i} X, \nu\right) \\
& =\widetilde{g}\left(f_{i} X+h_{i} X, \nu\right)+\widetilde{g}\left(\xi_{i}, \nabla \frac{\perp}{X} \nu-A_{\nu} X-\sum_{i=1}^{m} c_{i} g\left(h_{i} X, \nu\right) \xi_{i}\right) \\
& =\widetilde{g}\left(D_{X} \xi_{i}, \nu\right)+\widetilde{g}\left(\xi_{i}, D_{X} \nu\right) .
\end{aligned}
$$

By bilinearity, we get the property for any sections $\alpha$ and $\beta$ of $F$.

Now, we consider the curvature tensor associated with the connection $D$, denoted by $\mathcal{R}^{D}$ and defined by $\mathcal{R}^{D}(X, Y)=D_{X} D_{Y}-D_{Y} D_{X}-D_{[X, Y]}$. We can prove the following

Lemma 3.4. The connection $D$ is flat, that is, $\mathcal{R}^{D}=0$.

Proof: Let $X, Y, Z \in \Gamma(T M)$ and $\nu \in \Gamma(E)$. We will prove that $\mathcal{R}^{D}(X, Y) Z=0, \mathcal{R}^{D}(X, Y) \nu=$ 0 and $\mathcal{R}^{D}(X, Y) \xi_{i}=0$ for any $i \in\{1, \cdots, m\}$. Then by linearity of the curvature $\mathcal{R}^{D}$ in its third argument, we will get that $\mathcal{R}^{D}=0$. First, we have

$$
\begin{aligned}
D_{X} D_{Y} Z= & \nabla_{X} \nabla_{Y} Z+B\left(X, \nabla_{Y} Z\right)-\sum_{i=1}^{m} c_{i} g\left(f_{i} X, \nabla_{Y} Z\right) \xi_{i}+\nabla_{X}^{\perp} B(Y, Z)-A_{B(Y, Z)} X \\
& -\sum_{i=1}^{m} c_{i}\left[\bar{g}\left(B(Y, Z), h_{i} X\right) \xi_{i}+\left(f_{i} X+h_{i} X\right)+g\left(\nabla_{X} f_{i} Y, Z\right) \xi_{i}+g\left(f_{i} Y, \nabla_{X} Z\right) \xi_{i}\right] .
\end{aligned}
$$

Therefore, we get

$$
\begin{aligned}
\mathcal{R}^{D}(X, Y) Z= & R(X, Y) Z-\sum_{i=1}^{m} c_{i}\left[g\left(f_{i} Y, Z\right) f_{i} X-g\left(f_{i} X, Z\right) f_{i} Y\right]-A_{B(Y, Z)} X+A_{B(X, Z)} Y \\
& +\left(\nabla_{X} B\right)(Y, Z)-\left(\nabla_{Y} B\right)(X, Z)-\sum_{i=1}^{m} c_{i}\left[g\left(f_{i} Y, Z\right) h_{i} X-g\left(f_{i} X, Z\right) h_{i} Y\right] \\
& -\sum_{i=1}^{m} c_{i}\left[g\left(\left(\nabla_{X} f_{i}\right) Y-A_{h_{i} Y} X+s_{i} B(X, Y), Z\right)\right] \\
& -\sum_{i=1}^{m} c_{i}\left[g\left(\left(\nabla_{Y} f_{i}\right) X-A_{h_{i} X} Y+s_{i} B(X, Y), Z\right)\right] \\
= & 0
\end{aligned}
$$


by using the Gauss equation (first line), the Codazzi equation (second line) and equation (9) (third and fourth lines). Similarly, we have

$$
\begin{aligned}
D_{X} D_{Y} Z \nu= & \nabla_{X}^{\perp} \nabla_{Y}^{\perp} \nu-\nabla_{X} A_{\nu} Y-B\left(X, A_{\nu} Y\right)-A_{\nabla_{X}^{\perp} \nu} Y \\
& +\sum_{i=1}^{m} c_{i}\left[g\left(f_{i} X, A_{\nu} Y\right) \xi_{i}+\bar{g}\left(\nu, h_{i} Y\right)\left(f_{i} X+h_{i} X\right)\right] \\
& -\sum_{i=1}^{m} c_{i}\left[\bar{g}\left(\nabla_{Y}^{\perp} \nu, h_{i} X\right)-\bar{g}\left(\nabla_{X}^{\perp} \nu, h_{i} Y\right) \bar{g}\left(\nu, \nabla_{X}^{\perp} h_{i} Y\right)\right] .
\end{aligned}
$$

And hence,

$$
\begin{aligned}
& \mathcal{R}^{D}(X, Y) \nu=R^{\perp}(X, Y) \nu-B\left(X, A_{\nu} Y\right)+B\left(Y, A_{\nu} X\right)-\sum_{i=1}^{m} c_{i}\left[\bar{g}\left(h_{i} Y, \nu\right) h_{i} X-\bar{g}\left(h_{i} X, \nu\right) h_{i} Y\right] \\
& +\nabla_{Y} A_{\nu} X+A_{\nabla_{\frac{1}{Y}} \nu} X-\nabla_{X} A_{\nu} Y-A_{\nabla_{X}} \nu Y-\sum_{i=1}^{m} c_{i}\left[\bar{g}\left(h_{i} Y, \nu\right) f_{i} X-\bar{g}\left(h_{i} X, \nu\right) f_{i} Y\right] \\
& +\sum_{i=1}^{m} c_{i}\left[g\left(f_{i} X, A_{\nu} Y\right)-\bar{g}\left(\nu,\left(\nabla_{Y} h_{i}\right) X\right)+\bar{g}\left(t_{i} B(X, Y), \nu\right)\right] \\
& +\sum_{i=1}^{m} c_{i}\left[g\left(f_{i} Y, A_{\nu} X\right)-\bar{g}\left(\nu,\left(\nabla_{X} h_{i}\right) Y\right)+\bar{g}\left(t_{i} B(X, Y), \nu\right)\right]
\end{aligned}
$$

The first line in the right hand side vanishes due to Ricci equation. The second line vanishes by Codazzi equation and the third and fourth lines vanish by using equation (10). Finally, for any $i \in\{1, \cdots, m\}$, we have

$$
\begin{aligned}
D_{X} D_{Y} \xi_{j}= & \nabla_{X} f_{j} Y+B\left(X, f_{j} Y\right)-\sum_{i=1}^{m} c_{i} g\left(f_{i} X, f_{i} Y\right) \xi_{i} \\
& +\nabla_{X}^{\perp} h_{j} Y-A_{h_{j} Y} X-\sum_{i=1}^{m} c_{i} g\left(h_{i} X, h_{i} Y\right) \xi_{i}
\end{aligned}
$$

Hence,

$$
\begin{aligned}
\mathcal{R}^{D}(X, Y) \xi_{j}= & \left(\nabla_{X} f_{j}\right) Y-A_{h_{j} Y} X-s_{j} B(X, Y)-\left(\nabla_{Y} f_{j}\right) X+A_{h_{j} X} Y+s_{j} B(X, Y) \\
& \nabla_{X}^{\perp} h_{j} Y+B\left(X, f_{j} Y\right)-t_{j} B(X, Y)-\nabla_{Y}^{\perp} h_{j} X-B\left(Y, f_{j} X\right)-t_{j} B(X, Y) \\
= & 0
\end{aligned}
$$

by equations (9) and (10). Thus, we get that the connection $D$ is flat.

We define now for any $i \in\{1, \cdots, m\}$ the map $\pi_{i}: F \longrightarrow F$ by

$$
\begin{gathered}
\pi_{i} X=f_{i} X+h_{i} X, \\
\pi_{i} \nu=s_{i} \nu+t_{i} \nu, \\
\pi_{i} \xi_{j}=\delta_{i}^{j} \xi_{j},
\end{gathered}
$$

for any $X \in \Gamma(T M)$ and any $\nu \in \Gamma(E)$. We have the following properties

Lemma 3.5. For any $i \in\{1, \cdots, m\}$, the map $\pi_{i}$ is symmetric with respect to $\widetilde{g}$, parallel with respect to $D$ and

$$
\text { (1) } \pi_{i} \circ \pi_{i}=\pi_{i}
$$


(2) $\sum_{i=1}^{m} \pi_{i}=I d_{F}$.

Proof: The symmetry is clear because of the symmetry of $f_{i}, t_{i}$ and the fact that $s_{i}$ is the dual of $h_{i}$. The fact that $\pi_{i}$ is $D$-parallel comes from the definition of $D$ and equations (5) to (12). Indeed, we have for $X, Y \in \Gamma(T M)$,

$$
\begin{aligned}
\left(D_{X} \pi_{i}\right) Y= & D_{X}\left(\pi_{i} Y\right)-\pi_{i}\left(D_{X} Y\right) \\
= & D_{X}\left(f_{i} Y+h_{i} Y\right)-\pi_{i}\left(\nabla_{X} Y+B(X, Y)-\sum_{k=1}^{m} c_{k} g\left(f_{k} X, Y\right) \xi_{k}\right) \\
= & \nabla_{X}\left(f_{i} Y\right)+B\left(X, f_{i} Y\right)-\sum_{k=1}^{m} c_{k} g\left(f_{k} X, f_{i} Y\right) \xi_{k} \\
& +\nabla_{X}\left(h_{i} Y\right)-A_{h_{i} Y} X-\sum_{k=1}^{m} c_{k} g\left(h_{k} X, h_{i} Y\right) \xi_{k} \\
& -f_{i}\left(\nabla_{X} Y\right)+h_{i}\left(\nabla_{X} Y\right)-s_{i} B(X, Y)-t_{i} B(X, Y)+c_{i} g\left(f_{i} X, Y\right) \xi_{i} .
\end{aligned}
$$

By the use of equations (9) and (10), we get

$$
\begin{aligned}
\left(D_{X} \pi_{i}\right) Y & =c_{i} g\left(f_{i} X, Y\right) \xi_{i}-\sum_{k=1}^{m} c_{k}\left[g\left(f_{k} X, f_{i} Y\right)+g\left(h_{k} X, h_{i} Y\right) \xi_{k}\right] \\
& =c_{i} g\left(f_{i} X, Y\right) \xi_{i}-\sum_{k=1}^{m} c_{k}\left[g\left(f_{i} \circ f_{k} X+s_{i} \circ h_{k} X, Y\right)\right] \\
& =0,
\end{aligned}
$$

since, by (5), we have $f_{i} \circ f_{k}+s_{i} \circ h_{k}=\delta_{i}^{k} f_{i}$. The computations are analogous for a section $\nu$ of $E$ or for one of the $\xi_{i}$.

The relation $\pi_{i} \circ \pi_{i}=\pi_{i}$ is obvious from the definition of $\pi_{i}$ and relations (5) to (8). Finally, we get immediately that $\sum_{i=1}^{m} \pi_{i}=I d_{F}$ from the definition and assumption (4).

We consider the subsets $F^{i}$ of $F$ defined by

$$
F^{i}=\left\{\alpha \in F \mid \pi_{j} \alpha=\delta_{i}^{j} \alpha \text { for any } j \in\{1, \cdots, m\}\right\} .
$$

Note that, since the $\pi_{i}$ are symmetric, then the subbundles $F^{i}$ are orthogonal with respect to $\widetilde{g}$. We finally need a last lemma

Lemma 3.6. For any $i \in\{1, \cdots, m\}$, there exists orthonormal $n_{i}+1$ parallel sections $\sigma_{1}^{i}, \cdots, \sigma_{n_{i}+1}^{i}$ of $F^{i}$.

Proof: Let $p$ be a point of $M$. For any $i \in\{1, \cdots, m\}$, let $\left\{v_{1}^{i}, \cdots, v_{n_{1}+1}^{i}\right\}$ be an orthonormal basis of $F_{i}^{p}$, which is of dimension $n_{i}+1$ by the assumption on the rank of $\pi_{i}$. Moreover, since $\xi_{i}$ clearly belongs to $F_{i}$, we can choose $v_{i}^{1}=\sqrt{\left|c_{i}\right|} \xi_{1}(p)$. Thus, we have $\widetilde{g}\left(v_{1}^{i}, v_{1}^{i}\right)=\operatorname{sign}\left(c_{i}\right)$ and $\widetilde{g}\left(v_{k}^{i}, v_{k}^{i}\right)=1$ for $k \in\left\{2, \cdots, n_{i}+1\right\}$. Since the $F^{i}$ are orthogonal, the set of all $v_{i}^{k}$ forms an orthogonal basis of $F_{p}$. Now, since the connection $D$ is flat and $M$ is simply connected, then for any $i \in\{1, \cdots, m\}$ there exists a family of parallel sections $\sigma_{1}^{i}, \cdots, \sigma_{n_{i}+1}^{i}$, such that $\sigma_{k}^{i}(p)=v_{k}^{i}$. Moreover, since $D$ is compatible with the metric $\widetilde{g}$, then the sections are orthonormal. Finally, since the maps $\pi$ are $D$-parallel, then, for any $i \in\{1, \cdots, m\}$ and any $k \in\left\{1, \cdots, n_{i}+1\right\}$, $\pi_{i}\left(\sigma_{k}^{i}\right)=\sigma_{k}^{i}$, that is $\sigma_{k}^{i}$ is a section of $F^{i}$. This concludes the proof of the lemma. 
We will construct now the isometric immersion from $M$ into $\widetilde{P}$. For this, we consider the following functions. For $i \in\{1, \cdots, m\}$ and $k \in\left\{1, \cdots, n_{i}+1\right\}$, let $\varphi_{k}^{i}$ be defined by

$$
\varphi_{k}^{i}=\widetilde{g}\left(\sigma_{k}^{i}, \xi_{i}\right) .
$$

The candidate for the isometric immersion is

$$
\varphi: M \longrightarrow \mathbb{E}_{1} \times \cdots \times \mathbb{E}_{m}
$$

where $\mathbb{E}_{i}$ is the Euclidean space $\mathbb{R}^{n_{i}+1}$ if $c_{i}>0$ and the Minkowski space $\mathbb{L}^{n_{i}+1}$ if $c_{i}<0$. We will show that the map $\varphi$ goes into $\widetilde{P} \subset \mathbb{E}_{1} \times \cdots \times \mathbb{E}_{m}$ and satisfies all the properties stated in Theorem 3.2

First, we have

$$
\begin{aligned}
\frac{1}{c_{i}}=\widetilde{g}\left(\xi_{i} \cdot \xi_{i}\right) & =\sum_{k=1}^{n_{i}+1} \widetilde{g}\left(\xi_{i}, \sigma_{k}^{i}\right)^{2} \widetilde{g}\left(\sigma_{k}^{i}, \sigma_{k}^{i}\right) \\
& =\operatorname{sign}\left(c_{i}\right) \widetilde{g}\left(\xi_{i}, \sigma_{1}^{i}\right)^{2}+\sum_{k=2}^{n_{i}+1} \widetilde{g}\left(\xi_{i}, \sigma_{k}^{i}\right)^{2} \\
& =\operatorname{sign}\left(c_{i}\right)\left(\varphi_{1}^{i}\right)^{2}+\sum_{k=2}^{n_{i}+1}\left(\varphi_{k}^{i}\right)^{2} .
\end{aligned}
$$

Thus, we get that $\left(\varphi_{1}^{i}, \cdots, \varphi_{n_{i}+1}^{i}\right) \in M_{i}$, the $n_{i}$-dimensional simply connected space form of curvature $c_{i}$, and so, $\varphi(M)$ lies in $\widetilde{P}$.

Now, we will show that $\varphi$ is an immersion. For this, let $p \in M$ and $v \in T_{p} M$ so that $\varphi_{*}(v)=0$. From the definition of $\varphi$, the fact that $\varphi_{*}(v)=0$ implies

$$
\widetilde{g}\left(\sigma_{k}^{i}(p), \pi_{i} v\right)=\widetilde{g}\left(v_{k}^{i}, \pi_{i} v\right)=0,
$$

for any $i \in\{1, \cdots, m\}$ and $k \in\left\{1, \cdots, n_{i}+1\right\}$. Since, for any $i,\left\{v_{k}^{i}\right\}$ is an orthonormal basis of $F_{p}^{i}$, we get that $\pi_{i} v=0$. Moreover, from Lemma 3.5. $\sum_{i=1}^{m} \pi_{i}=I d_{F}$, then $v=0$. This holds for any $p$ and any $v$, so we get that $\varphi$ is an immersion.

Moreover, for $v, w \in T_{p} M$, we have

$$
\begin{aligned}
\left\langle\varphi_{*}(v), \varphi_{*}(w)\right\rangle & =\sum_{i=1}^{m}\left(\operatorname{sign}\left(c_{i}\right) \widetilde{g}\left(\sigma_{1}^{i}, \pi_{i} v\right) \widetilde{g}\left(\sigma_{1}^{i}, \pi_{i} w\right)+\sum_{k=2}^{n_{i}+1} \widetilde{g}\left(\sigma_{k}^{i}, \pi_{i} v\right) \widetilde{g}\left(\sigma_{k}^{i}, \pi_{i} w\right)\right) \\
& =\sum_{i=1}^{m} \widetilde{g}\left(\pi_{i} v, \pi_{i} w\right) \\
& =\widetilde{g}(v, w),
\end{aligned}
$$

where $\langle\cdot, \cdot\rangle$ is the (pseudo)-Euclidean metric on $\mathbb{E}_{1} \times \cdots \times \mathbb{E}_{m}$. Hence, $\varphi$ is an isometric immersion from $M$ into $\widetilde{P}$.

Now, we define the following bundle isomorphism

$$
\Phi: F \longrightarrow T\left(\mathbb{E}_{1} \times \cdots \times \mathbb{E}_{m}\right)_{\mid \varphi(M)},
$$


by $\Phi\left(\sigma_{k}^{i}\right)=e_{k}^{i}$, where $\left\{e_{1}^{i}, \cdots, e_{n_{i}+1}^{i}\right\}$ is the canonical frame of $T \mathbb{E}_{i}$ restricted to $\varphi(M)$. For $X \in \Gamma(T M)$, we have

$$
\begin{aligned}
\Phi(X) & =\sum_{i=1}^{m} \sum_{k=1}^{n_{i}+1} \widetilde{g}\left(X, \sigma_{k}^{i}\right) e_{k}^{i} \\
& =\sum_{i=1}^{m} \sum_{k=1}^{n_{i}+1} \widetilde{g}\left(\pi_{i} X, \sigma_{k}^{i}\right) e_{k}^{i} \\
& =\varphi_{*}(X) .
\end{aligned}
$$

Moreover, for any $i \in\{1, \cdots, m\}$, we have

$$
\Phi\left(\xi_{i}\right)=\sum_{k=1}^{n_{i}+1} \widetilde{g}\left(\xi_{i}, \sigma_{k}^{i}\right) e_{k}^{i} .=\sum_{k=1}^{n_{i}+1} \varphi_{k}^{i} e_{k}^{i} .
$$

Hence, $\Phi\left(\xi_{i}\right)$ is the normal direction of $M_{i}$ in $\mathbb{E}_{i}$. Since $\Phi$ is an isometry of the fibers, we deduce that $\Phi(E)$ is the normal bundle $T^{\perp} \varphi(M)$ of $\varphi(M)$ in $\widetilde{P}$. We denote by $\widetilde{\varphi}$ the restriction of $\Phi$ to $E$. It is clear that $\widetilde{\varphi}$ is an isomorphism of vector bundles between $E$ and $T^{\perp} \varphi(M)$.

Since $\Phi$ sends the orthonormal parallel sections $\left\{\sigma_{k}^{i}\right\}$ of $F$ onto the orthonormal parallel sections $\left\{e_{k}^{i}\right\}$ of $\mathbb{E}_{1} \times \cdots \times \mathbb{E}_{m}$, we have

$$
\begin{gathered}
\Phi\left(D_{X} Y\right)=\nabla_{\varphi_{*}(X)}^{0} \varphi_{*}(Y), \\
\Phi\left(D_{X} \nu\right)=\nabla_{\varphi_{*}(X)}^{0} \widetilde{\varphi}(\nu), \\
\Phi\left(D_{X} \xi_{i}\right)=\nabla_{\varphi_{*}(X)}^{0} \Phi\left(\xi_{i}\right),
\end{gathered}
$$

where $\nabla^{0}$ is the Levi-Civita connection of $\mathbb{E}_{1} \times \cdots \times \mathbb{E}_{m}$.

For any $i \in\{1, \cdots, m\}$, we define the map $\widetilde{\pi}_{i}=\Phi \circ \pi_{i} \circ \Phi^{-1}$. From this definition, it is clear that $\widetilde{\pi}_{i}\left(e_{k}^{j}\right)=\delta_{i}^{j} e_{k}^{j}$. Then, it follows that the maps $\widetilde{\pi}_{i}$ are symmetric, parallel along $\varphi(M)$ and satisfy $\widetilde{\pi}_{i} \circ \widetilde{\pi}_{j}=\delta_{i}^{j} \widetilde{\pi}_{i}$ and $\sum_{i=1}^{m} \widetilde{\pi}_{i}=\operatorname{Id}_{T\left(\mathbb{E}_{1} \times \cdots \times \mathbb{E}_{m}\right)}$. Thus, it is clear that these maps are the restrictions on $\varphi(M)$ of the projections on each factor $T \mathbb{E}_{i}$ of $T\left(\mathbb{E}_{1} \times \cdots \times \mathbb{E}_{m}\right)$.

Moreover, from the definition of $\tilde{\pi}_{i}$, we deduce immediately that

$$
\tilde{\pi}_{i}\left(\varphi_{*} X\right)=\varphi_{*}\left(f_{i} X\right)+\Phi\left(h_{i} X\right),
$$

and

Indeed, we have

$$
\tilde{\pi}_{i}(\Phi \xi)=\varphi_{*}\left(s_{i} X\right)+\Phi\left(t_{i} \xi\right)
$$

$$
\tilde{\pi}_{i}\left(\varphi_{*} X\right)=\Phi\left(\pi_{i}(X)\right)=\Phi\left(f_{i} X+h_{i} X\right)=\varphi_{*}\left(f_{i} X\right)+\widetilde{\varphi}\left(h_{i} X\right),
$$

and

$$
\widetilde{\pi}_{i}(\widetilde{\varphi}(\nu))=\Phi\left(\pi_{i}(\nu)\right)=\Phi\left(s_{i} \nu+h_{i} \nu\right)=\varphi_{*}\left(s_{i} \nu\right)+\widetilde{\varphi}\left(t_{i} \nu\right) .
$$

Finally, we will prove that the second fundamental form is given by $B$ and the normal connection is given by $\bar{\nabla}$. From Equation (13), we have

$$
\begin{aligned}
\nabla_{\varphi_{*}(X)}^{0} \varphi_{*}(Y) & =\Phi\left(D_{X} Y\right) \\
& =\Phi\left(\nabla_{X} Y+B(X, Y)-\sum_{i=1}^{m} c_{i} g\left(f_{i} X, Y\right) \xi_{i}\right) \\
& =\varphi_{*}\left(\nabla_{X} Y\right)+\widetilde{\varphi}(B(X, Y))-\sum_{i=1}^{m} c_{i} g\left(f_{i} X, Y\right) \Phi\left(\xi_{i}\right) .
\end{aligned}
$$


Then the normal part in $T \widetilde{P}$ is $\widetilde{\varphi}(B(X, Y))$, which implies that the second fundamental form of the immersion $\varphi$ is $\widetilde{\varphi} \circ B$. Moreover, from Equation (14), we have

$$
\begin{aligned}
\nabla_{\varphi_{*}(X)}^{0} \widetilde{\varphi}(\nu) & =\Phi\left(D_{X} \nu\right) \\
& =\Phi\left(\bar{\nabla}_{X} \nu-A_{\nu} X-\sum_{i=1}^{m} c_{i} g\left(h_{i} X, \nu\right) \xi_{i}\right) \\
& =\widetilde{\varphi}\left(\bar{\nabla}_{X} \nu\right)+\varphi_{*}\left(A_{\nu} X\right)-\sum_{i=1}^{m} c_{i} g\left(h_{i} X, \nu\right) \Phi\left(\xi_{i}\right) .
\end{aligned}
$$

Thus, the normal part in $T \widetilde{P}$ is $\widetilde{\varphi}\left(\bar{\nabla}_{X} \nu\right)$ and we deduce that $\nabla_{X}^{\frac{1}{X}} \widetilde{\varphi}(\nu)=\widetilde{\varphi}\left(\bar{\nabla}_{X} \nu\right)$. Then, we get $\nabla^{\perp} \widetilde{\varphi}=\widetilde{\varphi} \bar{\nabla}$. This concludes the proof of the existence in Theorem 3.2

Now, we will prove the uniqueness of this isometric immersion up to an isometry of $\widetilde{P}$. This follows directly from the following proposition.

Proposition 3.7. Let $\varphi, \varphi^{\prime}: M \longrightarrow \widetilde{P}$ be two isometric immersions with respective normal bundles $E, E^{\prime}$ and second fundamental forms $B, B^{\prime}$. Let $f_{i}, h_{i}$ and $f_{i}^{\prime}, h_{i}^{\prime}$ be the (1,1)-tensors defined by (21) for $\varphi$ and $\varphi^{\prime}$ respectively. Assume that

i) $f_{i} X=f_{i}^{\prime} X$ for any $i \in\{1, \cdots, m\}$ and $X \in \Gamma(T M)$,

ii) there exists an isometry of vector bundles $\phi: E \longrightarrow E^{\prime}$ so that

$$
\begin{gathered}
\phi\left(h_{i} X\right)=h_{i}^{\prime} X, \\
\phi(B(X, Y))=B^{\prime}(X, Y), \\
\phi\left(\bar{\nabla}_{X} \nu\right)=\bar{\nabla}_{X}^{\prime} \phi(\nu),
\end{gathered}
$$

for any $i \in\{1, \cdots, m\}, X, Y \in \Gamma(T M)$ and $\nu \in E$.

Then, there exists an isometry $\alpha$ of $\widetilde{P}$ such that $\varphi^{\prime}=\alpha \circ \varphi$ and $\alpha_{* \mid E}=\phi$.

Proof: We give the complete proof for $c_{m} \neq 0$, the case $c_{m}$ can be proven with a minor modification.

As previously, we denote by $\left\{e_{k}^{i}\right\}$, for $i \in\{1, \cdots, m\}$ and $k \in\left\{1, \cdots, n_{i}+1\right\}$ the canonical frame of $\mathbb{E}_{1} \times \cdots \times \mathbb{E}_{m}$. Hence, we denote by $\varphi_{k}^{i}$ and $\left(\varphi^{\prime}\right)_{k}^{i}$ the components of $\varphi$ and $\varphi^{\prime}$ respectively in the frame $\left\{e_{k}^{i}\right\}$. We consider the map $G: M \longrightarrow G L\left(\mathbb{E}_{1} \times \cdots \times \mathbb{E}_{m}\right)$ defined by

$$
\begin{gathered}
G_{p}\left(\varphi_{*}(X)\right)=\varphi_{*}^{\prime}(X), \\
G_{p}(\nu)=\phi(\nu), \\
G_{p}\left(\xi_{i}\right)=\xi_{i}^{\prime},
\end{gathered}
$$

for any $i \in\{1, \cdots, m\}, X, Y \in \Gamma(T M)$ and $\nu \in E$, and where $\xi_{i}$ and $\xi_{i}^{\prime}$ are defined by

$$
\xi_{i}=\sum_{k=1}^{n_{i}+1} \varphi_{k}^{i} e_{k}^{i}
$$

and

$$
\xi_{i}^{\prime}=\sum_{k=1}^{n_{i}+1}\left(\varphi^{\prime}\right)_{k}^{i} e_{k}^{i} .
$$

We will show that the map $G$ is constant, that is, that it does not depend on the point $p$. First of all, we remark that for any $i$ and any $X \in \Gamma(T M)$, we have $\nabla_{X}^{0} \xi_{i}=\pi_{i}\left(\varphi_{*}(X)\right)$, where $\pi_{i}$ is the projection on $T \mathbb{E}_{i}$ and $\nabla^{0}$ is the Levi-Civita connection of $\mathbb{E}_{1} \times \cdots \times \mathbb{E}_{m}$. Now, we will 
show that $\nabla^{0} G=0$, or equivalently that $\nabla_{X}^{0}(G(V))-G\left(\nabla_{X}^{0} V\right)=0$ for any $X \in \Gamma(T M)$ and $V \in \Gamma\left(T\left(\mathbb{E}_{1} \times \cdots \times \mathbb{E}_{m}\right)_{\mid \varphi(M)}\right)$.

First, for $V \in \varphi_{*}(T M)$, that is, $V=\varphi_{*}(Y)$ with $Y$ tangent to $M$, we have

$$
\begin{aligned}
\nabla_{X}^{0}\left(G\left(\varphi_{*}(Y)\right)\right)-G\left(\nabla_{X}^{0} \varphi_{*}(Y)\right)= & \nabla_{X}^{0} \varphi_{*}^{\prime}(Y)-G\left(\varphi_{*}\left(\nabla_{X} Y\right)+B(X, Y)\right) \\
& +\sum_{i=1}^{m} c_{i} g\left(f_{i}(X), Y\right) \xi_{i}^{\prime} \\
= & \varphi_{*}^{\prime}\left(\nabla_{X} Y\right)+B^{\prime}(X, Y)-\sum_{i=1}^{m} c_{i} g\left(f_{i}^{\prime}(X), Y\right) \xi_{i}^{\prime} \\
& -G\left(\varphi_{*}\left(\nabla_{X} Y\right)+B(X, Y)\right)+\sum_{i=1}^{m} c_{i} g\left(f_{i}(X), Y\right) \xi_{i}^{\prime} \\
= & 0,
\end{aligned}
$$

since, by assumption, $f_{i} X=f_{i}^{\prime} X$ and $\phi(B(X, Y))=B^{\prime}(X, Y)$. Now, if $\nu \in E$, we have

$$
\begin{aligned}
\nabla_{X}^{0}(G(\nu))-G\left(\nabla_{X}^{0} \nu\right)= & -A_{\phi(\nu)}^{\prime} X+\bar{\nabla}_{X}^{\prime} \phi(\nu)-\sum_{i=1}^{m} c_{i} \widetilde{g}\left(\phi(\nu), h_{i}^{\prime}(X)\right) \xi^{\prime} \\
& -G\left(-A_{\nu} X+\bar{\nabla}_{X} \nu-\sum_{i=1}^{m} c_{i} \widetilde{g}\left(\phi(\nu), h_{i}(X)\right) \xi\right) \\
= & 0,
\end{aligned}
$$

since by assumption, $\phi(B(X, Y))=B^{\prime}(X, Y), \phi\left(h_{i} X\right)=h_{i}^{\prime} X$ and $\phi\left(\bar{\nabla}_{X} \nu\right)=\bar{\nabla}_{X}^{\prime} \phi(\nu)$.

Finally, we have

$$
\begin{aligned}
\nabla_{X}^{0}(G(\xi))-G\left(\nabla_{X}^{0} \xi\right) & =\pi_{i}\left(\varphi_{*}^{\prime}(X)\right)-G\left(\pi_{i}\left(\varphi_{*}(X)\right)\right) \\
& =\varphi_{*}^{\prime}\left(f_{i}^{\prime} X\right)+h_{i}^{\prime} X-G\left(\varphi_{*}\left(f_{i} X\right)+h_{i} X\right) \\
& =0
\end{aligned}
$$

since $f_{i} X=f_{i}^{\prime} X$ and $\phi\left(h_{i} X\right)=h_{i}^{\prime} X$.

Hence, we get that the map $G$ is constant along $M$.

Uniqueness up to rigid motion is proved, which concludes the proof of Theorem 3.2 .

\section{Associated families of minimal SURFACes and Pluriminimal Kälher hyPersufaces}

In this section, we use Theorem 3.2 to prove the existence of associated families of minimal surfaces into the multiproduct $\widetilde{P}$.

Let $(\Sigma, g)$ be an oriented Riemannian surface. We denote by $J$ its complex structure, that is, the rotation of angle $\frac{\pi}{2}$ on $T M$. For any $\theta \in \mathbb{R}$, we set $\mathcal{R}_{\theta}=\cos (\theta) I+\sin (\theta) J$. Remark, that $\mathcal{R}_{\theta}$ is parallel. First, we have the following proposition.

Proposition 4.1. Assume that $\left(\Sigma, g, E, \bar{g}, \bar{\nabla}, B, f_{i}, h_{i}, t_{i}\right)$ satisfies the compatibility equation for $\widetilde{P}$ and that $B$ is trace-free for any $\xi \in E$, then $\left(\Sigma, g, E, \bar{g}, \bar{\nabla}, B_{\theta}, f_{i, \theta}, h_{i, \theta}, t_{, \theta}\right)$ also satisfies the 
compatibility equations for $\widetilde{P}$, where

$$
\begin{aligned}
& B_{\theta}(X, Y)=B\left(\mathcal{R}_{\theta} X, Y\right), \\
& f_{i, \theta}=\mathcal{R}_{\theta} \circ f_{i} \circ \mathcal{R}_{\theta}^{-1}, \\
& h_{i, \theta}=h_{i} \circ \mathcal{R}_{\theta}^{-1}, \\
& t_{i, \theta}=t_{i} .
\end{aligned}
$$

Moreover, $B_{\theta}$ is also trace-free for any $\xi \in E$.

Proof: First, from the definition of $f_{i, \theta}, h_{i, \theta}$ and $t_{i, \theta}$ and the fact that

$$
\begin{aligned}
& f_{i} \circ f_{j}+s_{i} \circ h_{j}=\delta_{i}^{j} f_{i}, \\
& t_{i} \circ t_{j}+h_{i} \circ s_{j}=\delta_{i}^{j} t_{i}, \\
& f_{i} \circ s_{j}+s_{i} \circ t_{j}=\delta_{i}^{j} s_{i}, \\
& h_{i} \circ f_{j}+t_{i} \circ h_{j}=\delta_{i}^{j} h_{i},
\end{aligned}
$$

we get immediately that $f_{i, \theta}$ and $t_{i, \theta}$ are symmetric and

$$
\begin{aligned}
& f_{i, \theta} \circ f_{j, \theta}+s_{i, \theta} \circ h_{j, \theta}=\delta_{i}^{j} f_{i, \theta}, \\
& t_{i, \theta} \circ t_{j, \theta}+h_{i, \theta} \circ s_{j, \theta}=\delta_{i}^{j} t_{i, \theta}, \\
& f_{i, \theta} \circ s_{j, \theta}+s_{i, \theta} \circ t_{j, \theta}=\delta_{i}^{j} s_{i, \theta}, \\
& h_{i, \theta} \circ f_{j, \theta}+t_{i, \theta} \circ h_{j, \theta}=\delta_{i}^{j} h_{i, \theta} .
\end{aligned}
$$

It is also clear that with this definition, the rank of $\pi_{i, \theta}$ is the same that the rank of $\pi_{i}$ and that

$$
\sum_{i=1}^{m} f_{i, \theta}=I d_{T M}, \quad \sum_{i=1}^{m} t_{i, \theta}=I d_{E} \quad \text { and } \quad \sum_{i=1}^{m} h_{i, \theta}=0
$$

Now, we will show that analogues of Equations (5)-(7) are satisfied for $\left(\Sigma, g, E, \bar{g}, \bar{\nabla}, B_{\theta}, f_{i, \theta}, h_{i, \theta}, t_{, \theta}\right)$. First, we have for $X, Y$ tangent to $\Sigma$

$$
\begin{aligned}
\nabla_{X} f_{i, \theta} Y-f_{i, \theta}\left(\nabla_{X} Y\right) & =\nabla_{X}\left(\mathcal{R}_{\theta} f_{i} \mathcal{R}_{\theta}^{-1} Y\right)-\mathcal{R}_{\theta} f_{i} \mathcal{R}_{\theta}^{-1}\left(\nabla_{X} Y\right) \\
& =\mathcal{R}_{\theta} \nabla_{X}\left(f_{i} \mathcal{R}_{\theta}^{-1} Y\right)-\mathcal{R}_{\theta} f_{i} \mathcal{R}_{\theta}^{-1}\left(\nabla_{X} Y\right)
\end{aligned}
$$

since $\mathcal{R}_{\theta}$ is parallel. Moreover, using (5), we get

$$
\begin{aligned}
\nabla_{X} f_{i, \theta} Y-f_{i, \theta}\left(\nabla_{X} Y\right)= & \mathcal{R}_{\theta} A_{h_{i}\left(\mathcal{R}_{\theta}^{-1} Y\right)} X+\mathcal{R}_{\theta} s_{i}\left(B\left(X, \mathcal{R}_{\theta}^{-1} Y\right)\right) \\
& +\mathcal{R}_{\theta} f_{i} \mathcal{R}_{\theta}^{-1}\left(\nabla_{X} Y\right)-\mathcal{R}_{\theta} f_{i}\left(\nabla_{X} \mathcal{R}_{\theta}^{-1} Y\right) \\
= & A_{h_{i, \theta}}^{\theta} X+s_{i, \theta}\left(B_{\theta}(X, Y)\right)
\end{aligned}
$$

which is the desired equation. The two other equations can be shown in a similar way. Finally, we prove that Gauss, Codazzi and Ricci equations are also fulfilled. 
First we consider the Gauss equation. We notice that, for a surface, we have

$$
\begin{aligned}
& \sum_{i=1}^{m} c_{i}\left[\left\langle f_{i, \theta} Y, Z\right\rangle f_{i, \theta} X-\left\langle f_{i, \theta} X, Z\right\rangle f_{i, \theta} Y\right]+A_{\theta B_{\theta}(Y, Z)} X-A_{\theta B_{\theta}(X, Z)} Y \\
& =\sum_{i=1}^{m} c_{i}\left[\mathcal{R}_{\theta} f_{i} \mathcal{R}_{\theta}^{-1} X \wedge \mathcal{R}_{\theta} f_{i} \mathcal{R}_{\theta}^{-1} Y\right] Z+\operatorname{det} A_{\theta} \\
& =\sum_{i=1}^{m} c_{i} \operatorname{det} \mathcal{R}_{\theta} f_{i} \mathcal{R}_{\theta}^{-1}+\operatorname{det} A_{\theta}=\sum_{i=1}^{m} c_{i} \operatorname{det} f_{i}+\operatorname{det} A=R(X, Y) Z
\end{aligned}
$$

since determinants are invariant under rotations. Hence Gauss equation is satisfied.

Let $\widetilde{\nabla}_{X} A_{\theta}^{\nu}=\nabla_{X} A_{\theta}^{\nu} Y-A_{\theta}^{\nu} \nabla_{X} Y-A_{\theta \nabla_{X}}^{\nu} \nu$. Considering now Codazzi equation, we have, using the property of $h_{i}$,

$$
\begin{aligned}
\left(\widetilde{\nabla}_{X} A_{\theta}^{\nu}\right) Y-\left(\widetilde{\nabla}_{Y} A_{\theta}^{\nu}\right) X & =\mathcal{R}_{\theta}\left[\left(\widetilde{\nabla}_{X} A^{\nu}\right) Y-\left(\widetilde{\nabla}_{Y} A^{\nu}\right) X\right] \\
& =\mathcal{R}_{\theta} \sum_{i=1}^{m} c_{i}\left[f_{i} Y\left\langle X, s_{i} \nu\right\rangle-f_{i} X\left\langle Y, s_{i} \nu\right\rangle\right] \\
& =\mathcal{R}_{\theta} \sum_{i=1}^{m} c_{i} f_{i}(X \wedge Y) s_{i} \nu=\sum_{i=1}^{m} c_{i} \mathcal{R}_{\theta} f_{i}(X \wedge Y) \mathcal{R}_{\theta}^{-1} \mathcal{R}_{\theta} s_{i} \nu \\
& =\sum_{i=1}^{m} c_{i}\left(\mathcal{R}_{\theta} f_{i} \mathcal{R}_{\theta}^{-1} X \wedge Y\right) s_{i, \theta} \nu=\sum_{i=1}^{m} c_{i}\left(f_{i, \theta} X \wedge Y\right) s_{i, \theta} \nu \\
& =\sum_{i=1}^{m} c_{i}\left[f_{i, \theta} Y\left\langle X, s_{i, \theta} \nu\right\rangle-f_{i, \theta} X\left\langle Y, s_{i, \theta} \nu\right\rangle\right]=\sum_{i=1}^{m} c_{i}\left[f_{i, \theta} Y\left\langle h_{i, \theta} X, \nu\right\rangle-f_{i, \theta} X\left\langle h_{i, \theta} Y, \nu\right\rangle\right]
\end{aligned}
$$

and Codazzi is satisfied.

Similarly we get for the Ricci equation, using the properties of the wedge product

$$
\begin{aligned}
R^{\perp}(X, Y) \xi & =\sum_{i=1}^{m} c_{i}\left[h_{i} \mathcal{R}_{\theta}^{-1} X \wedge h_{i} \mathcal{R}_{\theta}^{-1} Y\right] \xi+B_{\theta}\left(A_{\theta \xi} Y, X\right)-B_{\theta}\left(A_{\theta \xi} X, Y\right) \\
& =\sum_{i=1}^{m} c_{i}\left[h_{i} X \wedge h_{i} Y\right] \xi+B\left(A_{\xi} Y, X\right)-B\left(A_{\xi} X, Y\right)
\end{aligned}
$$

Since the surface is minimal, the shape operator anti-commutes with $J$ and we have indeed

$$
\begin{aligned}
B_{\theta}^{\nu}\left(A_{\theta \xi} Y, X\right)-B_{\theta}^{\nu}\left(A_{\theta \xi} X, Y\right) & =\left\langle\left[A_{\theta \nu}, A_{\theta \xi}\right] X, Y\right\rangle=\left\langle\left(\mathcal{R}_{\theta} A_{\nu} \mathcal{R}_{\theta} A_{\xi}-\mathcal{R}_{\theta} A_{\xi} \mathcal{R}_{\theta} A_{\nu}\right) X, Y\right\rangle \\
& =\left\langle\left(A_{\nu} \mathcal{R}_{\theta}^{-1} \mathcal{R}_{\theta} A_{\xi}-A_{\xi} \mathcal{R}_{\theta}^{-1} \mathcal{R}_{\theta} A_{\nu}\right) X, Y\right\rangle=\left\langle\left[A_{\nu}, A_{\xi}\right] X, Y\right\rangle .
\end{aligned}
$$

Finally, let $\left(e_{1}, e_{2}=J e_{1}\right)$ be a local orthonromal frame of $\Sigma$. We have

$$
\begin{aligned}
\operatorname{tr}\left(B_{\theta}\right)=B_{\theta}\left(e_{1}, e_{1}\right)+B_{\theta}\left(e_{2}, e_{2}\right) & =B\left(\mathcal{R}_{\theta} e_{1}, e_{1}\right)+B\left(\mathcal{R}_{\theta} e_{2}, e_{2}\right) \\
& =\cos \theta\left[B\left(e_{1}, e_{1}\right)+B\left(e_{2}, e_{2}\right)\right]=0
\end{aligned}
$$

since $B$ is trace-free.

From this proposition, we can prove easily the following theorem about associated families of minimal surfaces in multiproducts. Namely, we get the following statement. 
Theorem 4.2. Let $\Sigma$ be a simply connected surface and $x: M \longrightarrow \widetilde{P}$ be a conformal minimal immersion with normal bundle $E$, second fundamental form $B$ and normal connection $\nabla^{\perp}$. Let $f_{i}, h_{i}, s_{i}$ and $t_{i}$ be the $(1,1)$-tensors induced by the projections $\pi_{i}$. Let $p_{0} \in \Sigma$. Then, there exists a unique family $\left(x_{\theta}\right)_{\theta \in \mathbb{R}}$ of conformal minimal immersions $x_{\theta}: \Sigma \longrightarrow \widetilde{P}$ so that

i) $x_{\theta}\left(p_{0}\right)=x\left(p_{0}\right)$ and $d\left(x_{\theta}\right)_{p_{0}}=(d x)_{p_{0}}$,

ii) the metric induced by $X$ and $X_{\theta}$ are the same,

iii) the second fundamental form fo $x_{\theta}(\Sigma)$ in $\widetilde{P}$ is given by $B_{\theta}(X, Y)=B\left(R_{\theta} X, R_{\theta} Y\right)$, for any $X, Y \in \Gamma(T \Sigma)$.

iv) for any $i \in\{1, \cdots, m\}, X \in \Gamma(T \Sigma)$ and $\xi \in \Gamma(E)$,

$$
\pi_{i}\left(d x_{\theta} X\right)=d x_{\theta}\left(f_{i, \theta} X\right)+h_{i, \theta} X \quad \text { and } \quad \pi_{i}(\xi)=d x_{\theta}\left(s_{i, \theta} X\right)+t_{i, \theta} X
$$

Moreover, $x_{0}=x$ and the family $\left(x_{\theta}\right)_{\theta \in \mathbb{R}}$ is continuous with respect to $\theta$.

Proof: We just proved that $\left(\Sigma, g, E, \bar{g}, \bar{\nabla}, B_{\theta}, f_{i, \theta}, h_{i, \theta}, t_{, \theta}\right)$ satisfies the compatibility equations for each $\theta$. The theorem is then a direct consequence of theorem 3.2. The continuity is ensured by the construction of Theorem 3.2 .

\section{SURFACES IN $\mathbb{S}^{2} \times \mathbb{S}^{2}$}

Let $J$ be the complex structure on $\mathbb{S}^{2}$. We consider the following complex structures on $\mathbb{S}^{2} \times \mathbb{S}^{2}$

$$
J_{1}=(J, J), \quad J_{2}=(J,-J) .
$$

Obviously $J_{1}$ and $J_{2}$ commute with each other and the projection $\pi_{1}$ and $\pi_{2}$ on each of the factors are given by

$$
\pi_{1}=\frac{\mathrm{Id}+J_{1} J_{2}}{2}, \quad \pi_{2}=\frac{\mathrm{Id}-J_{1} J_{2}}{2} .
$$

From equation (11) we get

$$
\widetilde{R}(X, Y) Z=[\langle Y, Z\rangle X-\langle X, Z\rangle Y]+\left[\left\langle J_{1} Y, J_{2} Z\right\rangle J_{1} J_{2} X-\left\langle J_{1} X, J_{2} Z\right\rangle J_{1} J_{2} Y\right] .
$$

Let now $\Sigma$ be a surface isometrically immersed into $\mathbb{S}^{2} \times \mathbb{S}^{2}$. For $i \in\{1,2\}$, we define four operators $j_{i}: T \Sigma \rightarrow T \Sigma, k_{i}: T \Sigma \rightarrow N \Sigma, l_{i}: N \Sigma \rightarrow T \Sigma$ and $m_{i}: N \Sigma \rightarrow N \Sigma$ such that $J_{i}=j_{i}+k_{i}+l_{i}+m_{i}$.

From $J_{1} J_{2}=J_{2} J_{1}$ we get the following equations

$$
\begin{aligned}
& j_{1} j_{2}+l_{1} k_{2}=j_{2} j_{1}+l_{2} k_{1}, \\
& k_{1} j_{2}+m_{1} k_{2}=k_{2} j_{1}+m_{2} k_{1}, \\
& j_{1} l_{2}+l_{1} m_{2}=j_{2} l_{1}+l_{2} m_{1}, \\
& k_{1} l_{2}+m_{1} m_{2}=k_{2} l_{1}+m_{2} m_{1} .
\end{aligned}
$$

The property $J_{i}^{2}=-$ Id yields

$$
\begin{aligned}
& j_{i}^{2}+l_{i} k_{i}=-\operatorname{Id}_{T \Sigma}, \\
& k_{i} j_{i}+m_{i} k_{i}=0, \\
& j_{i} l_{i}+l_{i} m_{i}=0, \\
& k_{i} l_{i}+m_{i}^{2}=-\operatorname{Id}_{N \Sigma} .
\end{aligned}
$$


Moreover the fact that the operators $J_{i}$ are antisymmetric implies the antisymmetry of the operators $j_{i}$ as well as the property $\left\langle k_{i} X, \nu\right\rangle=-\left\langle X, l_{i} \nu\right\rangle$.

The parallelity of $J_{i}$ gives

$$
\begin{aligned}
& \nabla_{X}\left(j_{i} Y\right)-j_{i}\left(\nabla_{X} Y\right)=A_{k_{i} Y} X+l_{i}(B(X, Y)), \\
& \nabla_{X}^{\perp}\left(k_{i} Y\right)-k_{i}\left(\nabla_{X} Y\right)=m_{i}(B(X, Y))-B\left(X, j_{i} Y\right), \\
& \nabla_{X}^{\perp}\left(m_{i} \xi\right)-m_{i}\left(\nabla_{X}^{\perp} \xi\right)=-B\left(l_{i} \xi, X\right)-k_{i}\left(A_{\xi} X\right), \\
& \nabla_{X}\left(l_{i} \xi\right)-l_{i}\left(\nabla_{X}^{\perp} \xi\right)=-j_{i}\left(A_{\xi} X\right)+A_{m_{i} \xi} X .
\end{aligned}
$$

Finally, from (16), we get the Gauss equation

$$
\begin{aligned}
K= & \frac{1}{2}\left[1+\left(\left\langle j_{1} e_{1}, j_{2} e_{2}\right\rangle+\left\langle k_{1} e_{1}, k_{2} e_{2}\right\rangle\right)\left(\left\langle j_{1} e_{2}, j_{2} e_{1}\right\rangle+\left\langle k_{1} e_{2}, k_{2} e_{1}\right\rangle\right)\right. \\
& \left.-\left(\left\langle j_{1} e_{1}, j_{2} e_{1}\right\rangle+\left\langle k_{1} e_{1}, k_{2} e_{1}\right\rangle\right)\left(\left\langle j_{1} e_{2}, j_{2} e_{2}\right\rangle-\left\langle k_{1} e_{2}, k_{2} e_{2}\right\rangle\right)\right] \\
& +2|H|^{2}-\frac{|B|^{2}}{2}
\end{aligned}
$$

the Codazzi equation

$$
\begin{aligned}
& \left(\nabla_{X} B\right)(Y, Z)-\left(\nabla_{Y} B\right)(X, Z)= \\
& \quad \frac{1}{2}\left[\left\langle Y,\left(j_{1} j_{2}+l_{1} k_{2}\right) Z\right\rangle\left(k_{1} j_{2}+m_{1} k_{2}\right) X-\left\langle X,\left(j_{1} j_{2}+l_{1} k_{2}\right) Z\right\rangle\left(k_{1} j_{2}+m_{1} k_{2}\right) Y\right],
\end{aligned}
$$

and the Ricci equation

$$
\begin{aligned}
K^{\perp}= & {\left[\left\langle k_{1} j_{2}+m_{1} k_{2}\right) e_{2}, \nu_{1}\right\rangle\left\langle\left(k_{1} j_{2}+m_{1} k_{2}\right) e_{1}, \nu_{2}\right\rangle } \\
& \left.\left.\left\langle k_{1} j_{2}+m_{1} k_{2}\right) e_{1}, \nu_{1}\right\rangle\left\langle\left(k_{1} j_{2}+m_{1} k_{2}\right) e_{2}, \nu_{2}\right\rangle\right] \\
& \left.+\left\langle\left[A_{\nu_{2}}, A_{\nu_{1}}\right] e_{1}, e_{2}\right]\right\rangle .
\end{aligned}
$$

Remark 5.1. In [11] the Gauss, Codazzi and Ricci equations are expressed with the help of the two Kähler functions $C_{1}$ and $C_{2}: \Sigma \rightarrow \mathbb{R}$ defined by $\varphi^{*} \omega_{i}=C_{i} \omega_{\Sigma}, i=1,2$, with $\omega_{\Sigma}$ the area form on $\Sigma$. A tidious but straightforward computation shows that those two formulations are equivalent.

Now, we are able to reformulate the main theorem in the case of $\mathbb{S}^{2} \times \mathbb{S}^{2}$ in terms of complex structures instead of projections on each factor.

Corollary 5.2. Let $\left(\Sigma^{2}, g\right)$ be a Riemannian surface and $\left(E,\langle\cdot, \cdot\rangle_{E}, \nabla^{E}\right)$ a rank 2 vector bundle over $\Sigma$ endowed with a scalar product and a compatible connection. Suppose that there exists a symmetric (2,1)-tensor field $B: T \Sigma \times T \Sigma \rightarrow E$ and eight operators $j_{i}: T \Sigma \rightarrow T \Sigma, k_{i}: T \Sigma \rightarrow E$, $l_{i}: E \rightarrow T \Sigma$ and $m_{i}: E \rightarrow E, i=1,2$ satisfying conditions (17) to (28) and the Gauss, Codazzi and Ricci equations (29), (30) and (31). Then, there exists a unique (up to isometries of $\mathbb{S}^{2} \times \mathbb{S}^{2}$ ) isometric immersion from $\Sigma$ into $\mathbb{S}^{2} \times \mathbb{S}^{2}$ with $E$ as normal bundle, $B$ as second fundamental form and such that the restrictions of the complex structures $J_{i}$ over $\Sigma$ are given by $j_{i}, k_{i}, l_{i}$ and $m_{i}$. 
Proof: Define the following operators

$$
\begin{aligned}
f_{1} & =\operatorname{Id}_{T \Sigma}+j_{1} j_{2}+l_{1} k_{2}, \quad f_{2}=\operatorname{Id}_{T \Sigma}-j_{1} j_{2}-l_{1} k_{2} \\
h_{1} & =k_{1} j_{2}+m_{1} k_{2},=-h_{2}, \\
s_{1} & =j_{1} l_{2}+l_{1} m_{2}=-s_{2} \\
t_{1} & =\operatorname{Id}_{E}+k_{1} l_{2}+m_{1} m_{2}, \quad t_{2}=\operatorname{Id}_{E}-k_{1} l_{2}-m_{1} m_{2} .
\end{aligned}
$$

We can show easily that equations (17) to (31) imply that these operators satisfy the compatibility equations for $\mathbb{S}^{2} \times \mathbb{S}^{2}$ given by Definition 3.1. The conclusion follows easily from Theorem 3.2

Remark 5.3. We remind (see for example 11]) that an immersion $\varphi: \Sigma \longrightarrow \mathbb{S}^{2} \times \mathbb{S}^{2}$ is called complex if it is complex with respect to $J_{1}$ or $J_{2}$. It is called Lagrangian, if it is Lagrangian with respect to $J_{1}$ or $J_{2}$.

First Case: $\Sigma$ is a complex surface with respect to one of the complex structures $J_{i}$. Then it is automatically minimal. Moreover $k_{i}=l_{i}=0, j_{i}$ and $m_{i}$ are parallel complex structures on $T \Sigma$ and $N \Sigma$ respectively, and $j_{1}$ commutes with $j_{2}$, as well as $m_{1}$ with $m_{2}$. Assume without loss of generality that $\Sigma$ is complex with respect to $J_{1}$, then the Gauss, Codazzi and Ricci equations simplify to

$$
\begin{aligned}
& K=\frac{1}{2}\left[1+\left\langle j_{1} e_{1}, j_{2} e_{2}\right\rangle\left\langle j_{1} e_{2}, j_{2} e_{1}\right\rangle-\left\langle j_{1} e_{1}, j_{2} e_{1}\right\rangle\left\langle j_{1} e_{2}, j_{2} e_{2}\right\rangle\right]-\frac{|B|^{2}}{2} \\
& \left(\nabla_{X} B\right)(Y, Z)-\left(\nabla_{Y} B\right)(X, Z)=\frac{1}{2}\left[\left\langle Y, j_{1} j_{2} Z\right\rangle m_{1} k_{2} X-\left\langle X, j_{1} j_{2} Z\right\rangle m_{1} k_{2} Y\right] \\
& \left.K^{\perp}=\left[\left\langle m_{1} k_{2} e_{2}, \nu_{1}\right\rangle\left\langle m_{1} k_{2} e_{1}, \nu_{2}\right\rangle-\left\langle m_{1} k_{2} e_{1}, \nu_{1}\right\rangle\left\langle m_{1} k_{2} e_{2}, \nu_{2}\right\rangle\right]+\left\langle\left[A_{\nu_{2}}, A_{\nu_{1}}\right] e_{1}, e_{2}\right]\right\rangle .
\end{aligned}
$$

Notice that the only examples of complex surfaces with respect to both complex structures $J_{1}$ and $J_{2}$ are slices $\mathbb{S}^{2} \times p=\left\{(x, p) \in \mathbb{S}^{2} \times \mathbb{S}^{2} \mid x \in \mathbb{S}^{2}\right\}$ and $p \times \mathbb{S}^{2}=\left\{(p, x) \in \mathbb{S}^{2} \times \mathbb{S}^{2} \mid x \in \mathbb{S}^{2}\right\}$.

Second Case: $\Sigma$ is Lagrangian with respect to $J_{i}$, then $j_{i}=m_{i}=0$. Assuming again without loss of generality that $\Sigma$ is Lagrangian with respect to $J_{1}$, the Gauss, Codazzi and Ricci equations simplify in the following way

$$
\begin{aligned}
& K=\frac{1}{2}\left[1-\left\langle k_{1} e_{1}, k_{2} e_{2}\right\rangle\left\langle k_{1} e_{2}, k_{2} e_{1}\right\rangle+\left\langle k_{1} e_{1}, k_{2} e_{1}\right\rangle\left\langle k_{1} e_{2}, k_{2} e_{2}\right\rangle\right]+2|H|^{2}-\frac{|B|^{2}}{2}, \\
& \left.\left(\nabla_{X} B\right)(Y, Z)-\left(\nabla_{Y} B\right)(X, Z)=\frac{1}{2}\left[\left\langle Y, l_{1} k_{2}\right) Z\right\rangle k_{1} j_{2} X-\left\langle X, l_{1} k_{2} Z\right\rangle k_{1} j_{2} Y\right], \\
& \left.K^{\perp}=\left[\left\langle k_{1} j_{2} e_{2}, \nu_{1}\right\rangle\left\langle k_{1} j_{2} e_{1}, \nu_{2}\right\rangle-\left\langle k_{1} j_{2} e_{1}, \nu_{1}\right\rangle\left\langle k_{1} j_{2} e_{2}, \nu_{2}\right\rangle\right]+\left\langle\left[A_{\nu_{2}}, A_{\nu_{1}}\right] e_{1}, e_{2}\right]\right\rangle .
\end{aligned}
$$

Notice that $\Sigma$ is Lagrangian for both $J_{1}$ and $J_{2}$ if and only if it is the product $\varphi(s, t)=(\alpha(s), \beta(t))$ of two curves $\alpha, \beta$ in $\mathbb{S}^{2}$. The Clifford torus is the only example of a minimal such surface.

Third Case: $\Sigma$ is Lagrangian with respect to $J_{1}$ (hence $j_{1}=m_{1}=0$ ) and complex with respect to $J_{2}$ (hence $k_{2}=l_{2}=0$ ).

$$
\begin{aligned}
& K=\frac{1}{2}-\frac{|B|^{2}}{2} \\
& \left(\nabla_{X} B\right)(Y, Z)-\left(\nabla_{Y} B\right)(X, Z)=0, \\
& \left.K^{\perp}=\left[\left\langle k_{1} j_{2} e_{2}, \nu_{1}\right\rangle\left\langle k_{1} j_{2} e_{1}, \nu_{2}\right\rangle-\left\langle k_{1} j_{2} e_{1}, \nu_{1}\right\rangle\left\langle k_{1} j_{2} e_{2}, \nu_{2}\right\rangle\right]+\left\langle\left[A_{\nu_{2}}, A_{\nu_{1}}\right] e_{1}, e_{2}\right]\right\rangle .
\end{aligned}
$$


The only example of such a surface is the diagonal $\mathbf{D}=\left\{(x, x) \in \mathbb{S}^{2} \times \mathbb{S}^{2} \mid x \in \mathbb{S}^{2}\right\}$.

\section{REFERENCES}

[1] O. Bonnet, Mémoire sur la théorie des surfaces applicables sur une surface donnée Journal de l'École Polytechnique, 41 (1865), 201-230 and 42 (1867), 1-151.

[2] B. Daniel, Isometric immersions into $\mathbb{S}^{n} \times \mathbb{R}$ and $\mathbb{H}^{n} \times \mathbb{R}$ and applications to minimal surfaces, Trans. Amer. Math. Soc. 361 (12) (2009), 6255-6282.

[3] B. Daniel, Isometric immersions into 3-dimensional homogeneous manifolds, Comment. Math. Helv. 82 (2007), 87-131.

[4] F. Dillen, Equivalence theorems in affine differential geometry, Geom. Dedicata 32, 1 (1989), 81-92.

[5] F. Dillen, K. Nomizu \& L. Vranken, Conjugate connections and Radons theorem in affine differential geometry. Monatsh. Math. 109 (3) (1990), 221-235.

[6] D. Kowalczyk, Isometric immersions into products of space forms, Geom. Dedicata 151 (2011), 1-8.

[7] M.-A. Lawn \& M. Ortega, A Fundamental Theorem for Hypersurfaces in Semi-Riemannian Warped Products, J. Geom. Phys., to appear.

[8] J.H. Lira, R. Tojeiro \& F. Vitório, A Bonnet theorem for isometric immersions into products of space forms Arch. Math. (Basel) 95 (5) (2010), 469-479.

[9] J. Roth, Isometric immersion into Lorentzian products, Int. J. Geom. Method. Mod. Phys, 8 (2011) no.6, $1-22$.

[10] K. Tenenblat, On isometric immersions of Riemannian manifolds, Bol. Soc. Brasil. Mat. 2 (1971) no. 2, 23-36.

[11] F. Torralbo, F. Urbano Minimal surfaces in $\mathbb{S}^{2} \times \mathbb{S}^{2}$, J. Geom. Anal. to appear. 